\title{
Theory of a Slotted-Sphere Antenna Immersed in a Compressible Plasma. ${ }^{1}$ Part I.
}

\author{
James R. Wait \\ Contribution From the Central Radio Propagation Laboratory, National Bureau of \\ Standards, Boulder, Colo.
}

(Received May 20, 1964)

\begin{abstract}
Radiation from a finite source in a compressible electron plasma is considered. The theoretical model is a perfectly conducting sphere which is excited uniformly by an annular slot. The configuration is such that Maxwell's equations, when combined with a (singlefluid) continuum theory of fluid dynamies, are separable. It is shown that a portion of the total power supplied by the source is radiated as an acoustic wave in the electron fluid.
\end{abstract}

\section{Introduction}

When an antenna is placed in an ionized medium such as the upper atmosphere, energy may be coupled into acoustic-type waves. A theory which accounts for this phenomenon must take account of the finite compressibility of the electron fluid. There is some evidence [Whale, 1963, 1964] that rocket-borne antennas excite electron-acoustic waves in the ionosphere. Unfortunately, the situation is complicated by the presence of the earth's magnetic field and the existence of a plasma sheath around the antenna.

It is the purpose of this paper to consider an idealized model which should give some insight into the phenomenon of radiation in compressible plasma media. The earth's magnetic field is neglected throughout. The antenna model assumed here is a perfectly conducting sphere which is excited by a thin annular slot. The slotted-sphere antenna is assumed to be in intimate contact with the homogeneous plasma.

A number of related investigations of the stated problem have been published recently. A short review of this work is in order.

In a rather interesting note, Hessel and Shmoys [1962] consider the excitation of electromagnetic and plasma (acoustic) waves by a point current source in a homogeneous electron plasma of infinite extent. The plasma is assumed to be an ideal gas coupled to the electromagnetic field by the motion of the electrons. Collisions are neglected, the electron drift velocity is taken to be zero, and the static magnetic field is also neglected. For these conditions, they find the ratio of total radiated power $P_{p}$ in the acoustic wave to that of $P_{e}$ in the electromagnetic wave to be given by

$$
P_{p} / P_{e}=(1 / 2)(c / u)^{3}\left(\omega_{\mathrm{o}} / \omega\right)^{2},
$$

where $c$ is the velocity of light, $u$ is the acoustic velocity, $\omega_{0}$ is the plasma frequency, and $\omega$ is the operating frequency. This formula suggests that for an ionized medium, most of the power goes into the acoustic mode since $u<<c$ in most cases. This is a rather startling result. Hessel and Shmoys [1962] then suggest that a prescribed current distribution on a rigid sphere would give quite different results.

A related problem has been treated by Chen [1963] who arrives at the same expression as above for the power ratio in the case of an infinitesimal electric current element immersed in the compressible plasma. He extends his results to a thin wire antenna of finite length.

1 The work was supported by the Air Force Cambridge Research Laboratories, Bedford, Mass., PR O-62-201. 
To facilitate the analysis, he assumes a sinusoidal current distribution with a propagation constant assumed to be identical to that for plane electromagnetic waves in the plasma. Chen then arrives at the remarkable result that the ratio of the powers in the electromagnetic and the plasma waves is independent of the velocity ratio $(u / c)$.

A fundamental investigation was carried out by Seshadri [1963a] who considered a magnetic line source immersed in a compressible plasma. The static magnetic field was taken to be parallel to the infinite line source. By examining the behavior of these waves at the limiting case of high frequency, they are identified as the usual plasma (acoustic) and electromagnetic waves.

The excitation of coupled electromagnetic and plasma (acoustic) waves by a dipole moving along the axis of a compressible plasma column has been considered by Yildiz and Samaddar [1963]. The solution of this problem contains an acoustical shock wave which may be called acoustical Cerenkov radiation.

The conversion of electromagnetic waves into acoustic waves at a vacuum-plasma interface has been considered by Hessel et al. [1962], Seshadri [1963b], Yildiz [1963], and Wait [1964] for various idealized models.

\section{General Equations}

The plasma medium is regarded to be a one-component electron fluid. That is, the ions are neglected in the equation of motion, yet their presence is required to neutralize electrically the plasma. It is also assumed that the amplitude of the plasma and electromagnetic oscillations are sufficiently small that linearized equations may be valid [Oster, 1960]. The average number density of electrons is denoted $n_{0}$ which is regarded as constant in the plasma region. The pressure deviation of the electrons from the mean is $p$ and their mean velocity is $\vec{v}$. As usual, the electric and magnetic fields are denoted $\vec{E}$ and $\vec{H}$, respectively.

The linearized hydrodynamic equation of motion is

$$
m n_{0} \frac{\partial \vec{v}}{\partial t}=n_{0} e \vec{E}-\nabla p
$$

where $e$ and $m$ are the charge and mass of the electron, respectively. The equation of continuity, combined with the equation of state, leads readily to

$$
u^{2} m n_{0} \nabla \cdot \vec{v}=-\frac{\partial p}{\partial t}
$$

where $u$ is the velocity of sound in the electron gas.

Maxwell's equations for the electromagnetic fields in the plasma are given by

$$
\nabla \times \vec{E}=-\mu_{0} \frac{\partial \vec{H}}{\partial t}
$$

and

$$
\nabla \times \vec{H}=\epsilon_{0} \frac{\partial \vec{E}}{\partial t}+n_{0} e \vec{v}
$$

where $\mu_{0}$ and $\epsilon_{0}$ are the magnetic permeability and dielectric constant of free space, respectively. Without subsequent loss of generality, all field quantities are assumed to vary as exp $(i \omega t)$ and, thus, the derivative $\partial / \partial t$ may be replaced by $i \omega$ everywhere.

Except where noted, collisions between particles and other dissipative effects are neglected in this paper. 


\section{Formulation in the Spherical Geometry}

The compressible plasma is taken to occupy the space exterior to a spherical region of radius $a$ which contains the source(s). Thus, in terms of spherical coordinates $(r, \theta, \phi)$, the tangential electric fields on the surface $r=a$ are to be prescribed. To simplify the algebra, it is assumed that the prescribed field has only an $E_{\theta}$ component which is independent of $\phi$. Thus, as may be verified below, the resultant magnetic field has only an $H_{\phi}$ component everywhere. Furthermore, the $E_{\phi}$ component of the field and the $\phi$ component of the velocity are identically zero everywhere. The resulting electromagnetic field may be described as transverse magnetic or simply TM. The continuity equation for the plasma is thus given by

$$
\frac{u^{2} m n_{0}}{r^{2} \sin \theta}\left[\sin \theta \frac{\partial}{\partial r}\left(r^{2} v_{r}\right)+r \frac{\partial}{\partial \theta}\left(v_{\theta} \sin \theta\right)\right]=-i \omega p
$$

while the equations of motion for the plasma are

$$
i \omega m n_{0} v_{r}=n_{0} e E_{r}-\frac{\partial p}{\partial r}
$$

and

$$
i \omega m n_{0} v_{\theta}=n_{0} e E_{\theta}-\frac{1}{r} \frac{\partial p}{\partial \theta}
$$

Combining (6) and (7) with (4) leads readily to the set

$$
\begin{aligned}
& E_{\theta}=-\frac{1}{i \epsilon \omega} \frac{1}{r} \frac{\partial}{\partial r}\left(r H_{\phi}\right)-\frac{e}{m \epsilon \omega^{2} r} \frac{\partial p}{\partial \theta}, \\
& E_{r}=\frac{1}{i \epsilon \omega} \frac{1}{r \sin \theta} \frac{\partial}{\partial \theta}\left(\sin \theta H_{\phi}\right)-\frac{e}{m \epsilon \omega^{2}} \frac{\partial p}{\partial r}, \\
& v_{\theta}=\frac{e}{m \epsilon \omega^{2} r} \frac{\partial}{\partial r}\left(r H_{\phi}\right)-\frac{\epsilon_{0}}{i \epsilon \omega n_{0} m r} \frac{\partial p}{\partial \theta}, \\
& v_{r}=-\frac{e}{m \epsilon \omega^{2}} \frac{1}{r \sin \theta} \frac{\partial}{\partial \theta}\left(\sin \theta H_{\phi}\right)-\frac{\epsilon_{0}}{i \epsilon \omega n_{0} m} \frac{\partial p}{\partial r},
\end{aligned}
$$

where

$$
\frac{\epsilon}{\epsilon_{0}}=1-\frac{\omega_{0}^{2}}{\omega^{2}} \text { with } \omega_{0}^{2}=\frac{n_{0} e^{2}}{m \epsilon_{0}} .
$$

In the above, $\epsilon / \epsilon_{0}$ is the relative dielectric constant of the plasma while $\omega_{0}$ is the electron plasma frequency.

By combining Maxwell's equations (3) and (4) with the motion and continuity equations (5), (6), and (7), it is not difficult to show that for $r>a$,

and

$$
r \frac{\partial^{2}}{\partial r^{2}}\left(r H_{\phi}\right)+\frac{\partial}{\partial \theta}\left[\frac{1}{\sin \theta} \frac{\partial}{\partial \theta}\left(\sin \theta H_{\phi}\right)\right]+k_{e}^{2} r^{2} H_{\phi}=0
$$

$$
\frac{\partial}{\partial r}\left(r^{2} \frac{\partial p}{\partial r}\right)+\frac{1}{\sin \theta} \frac{\partial}{\partial \theta}\left(\sin \theta \frac{\partial p}{\partial \theta}\right)+k_{p}^{2} r^{2} p=0
$$

where

$$
k_{e}=\sqrt{\epsilon \mu_{0}} \omega=\frac{\omega}{c}\left(\frac{\epsilon}{\epsilon_{0}}\right)^{\frac{1}{2}}
$$

and

$$
k_{p}=\frac{\omega}{u}\left(\frac{\epsilon}{\epsilon_{0}}\right)^{\frac{1}{2}}
$$


Appropriate solutions of (12) and (13), which give rise to outgoing waves at infinity, are

and

$$
H_{\phi}=\sum_{n=1}^{\infty} \frac{\hat{K}_{n}\left(i k_{e} r\right)}{\left(i k_{e} r\right)} c_{n} P_{n}^{\prime}(\cos \theta)
$$

$$
p=\sum_{n=1}^{\infty} \frac{\hat{K}_{n}\left(i k_{p} r\right)}{\left(i k_{p} r\right)} a_{n} P_{n}(\cos \theta),
$$

where $c_{n}$ and $a_{n}$ are unknown coefficients, and where the summation is over integral values of $n$. In the above, $\hat{K}_{n}(z)$ is a spherical Bessel function which satisfies

$$
\frac{d^{2} \hat{K}_{n}(z)}{d z^{2}}=\left[1+\frac{n(n+1)}{z^{2}}\right] \hat{K}_{n}(z),
$$

while $P_{n}(\cos \theta)$ is a Legendre polynomial which satisfies

and, finally,

$$
\sin \theta \frac{d}{d \theta}\left(\sin \theta \frac{d P_{n}}{d \theta}\right)+n(n+1) \sin ^{2} \theta P_{n}=0,
$$

$$
P_{n}^{\prime}(\cos \theta)=\frac{d}{d \theta} P_{n}(\cos \theta)
$$

Following Schelkunoff [1943, 1952], the spherical function which satisfies (18) is taken to be

$$
\hat{K}_{n}(z)=e^{-z} \sum_{m=0}^{n} \frac{(n+m) !}{m !(n-m) !(2 z)^{m}} \cdot
$$

Thus, for $k_{e} r$ and $k_{p} r>>1$,

and

$$
\hat{K}_{n}\left(i k_{e} r\right) \sim e^{-i k_{e^{r}}}
$$

$$
\hat{K}_{n}\left(i k_{p} r\right) \sim e^{-i k_{p} r},
$$

which illustrates the outgoing character of the waves. It is also evident from the definitions of $k_{e}$ and $k_{p}$, given by (14) and (15), that radiation will not take place if $\epsilon / \epsilon_{0}<0$ (i.e., $\omega<\omega_{0}$ ). In what follows, it is assumed that $k_{e}$ and $k_{p}$ are both real, corresponding to $\epsilon / \epsilon_{0}>0$.

An explicit general expression for the $r$ component of the velocity $\vec{v}$ is obtained by using (11), (16), and (17), and getting an assist from (19). Thus, for $r \geqq a$

$$
v_{r}=\sum_{n=1}^{\infty}\left\{c_{n} \frac{e n(n+1)}{\epsilon \omega^{2} m r} \frac{\hat{K}_{n}\left(i k_{e} r\right)}{\left(i k_{e} r\right)}-a_{n} \frac{\epsilon_{0} k_{p}}{\epsilon \omega n_{0} m}\left[\frac{\hat{K}_{n}^{\prime}\left(i k_{p} r\right)}{i k_{p} r}-\frac{\hat{K}_{n}\left(i k_{p} r\right)}{\left(i k_{p} r\right)^{2}}\right]\right\} \times P_{n}(\cos \theta),
$$

where the prime indicates a derivative with respect to the argument of the Bessel function [i.e., $\hat{K}_{n}^{\prime}(z)=d \hat{K}_{n}(z) / d z$. The boundary condition imposed on the velocity is that $v_{r}=0$ at $r=a$. In other words, the spherical boundary is assumed to be rigid as far as the acoustic wave is considered. A simple calculation then leads to the result that

$$
\frac{a_{n}}{c_{n}}=n(n+1) \frac{e n_{0}}{\epsilon_{0} \omega a k_{p}} \frac{\left[\frac{\hat{K_{n}}\left(i k_{e} a\right)}{\left(i k_{e} a\right)}\right]}{\left[\frac{\hat{K_{n}}\left(i k_{p} a\right)}{\left(i k_{p} a\right)}-\frac{\hat{K}_{n}\left(i k_{p} a\right)}{\left(i k_{p} a\right)^{2}}\right]} \cdot
$$

The problem is formally solved when $a_{n}$ is determined by the specified conditions at the source. This task is deferred until later. 


\section{Radiated Power Considerations}

The total power $P$, radiated from the source, may be obtained by integrating the radial component of the Poynting vector over an infinitely large sphere surrounding the source. Thus,

$$
P=\operatorname{Lim}_{r \rightarrow \infty} \frac{1}{2} \int_{0}^{\pi}\left[E_{\theta} H_{\phi}^{*}+p v_{r}^{*}\right] 2 \pi r^{2} \sin \theta d \theta .
$$

The asymptotic form of the field components, valid for $k_{e} r>>1$, are obtained from (8), (9), (16), (17), and (22). Thus,

$$
H_{\phi} \sim \sum_{n=1}^{\infty} c_{n} \frac{e^{-i k_{e} r}}{i k_{e} r} P_{n}^{\prime}(\cos \theta)
$$

and

$$
E_{\theta} \sim-\eta_{e} \sum_{n=1}^{\infty} c_{n} \frac{e^{-i k_{e} r}}{i k_{e} r} P_{n}^{\prime}(\cos \theta)
$$

where $\eta_{e}=\mu_{0} \omega / k_{e}$. In a similar manner, by making use of (10), (11), (16), (17), and (23), it is found that

and

$$
p \sim \sum_{n=1}^{\infty} a_{n} \frac{e^{-i k_{p} r}}{i k_{p} r} P_{n}(\cos \theta)
$$

$$
v_{r} \sim-\frac{\epsilon_{0} k_{p}}{\epsilon \omega n_{0} m} \sum_{n=1}^{\infty} a_{n} \frac{e^{-i k_{p} r}}{i k_{p} r} P_{n}(\cos \theta) .
$$

The $\theta$ integration in (26) may now be readily carried out if use is made of the orthogonality relations [Morse and Feshbach, 1953]

$$
\begin{aligned}
\int_{0}^{\pi} P_{n}(\cos \theta) P_{m}(\cos \theta) \sin \theta d \theta & =\frac{2}{2 n+1} \quad \text { if } n=m \\
& =0 \quad \text { if } n \neq m,
\end{aligned}
$$

and

$$
\begin{aligned}
\int_{0}^{\pi} P_{n}^{\prime}(\cos \theta) P_{m}^{\prime}(\cos \theta) \sin \theta d \theta & =\frac{2 n(n+1)}{2 n+1} \quad \text { if } n=m \\
& =0 \quad \text { if } n \neq m .
\end{aligned}
$$

Thus, it is found readily that

$$
P=2 \pi \frac{\eta_{e}}{k_{e}^{2}} \sum_{n=1}^{\infty} \frac{n(n+1)}{(2 n+1)}\left|c_{n}\right|^{2}+2 \pi \frac{\epsilon_{0}}{k_{p} \epsilon \omega n_{0} m} \sum_{n=1}^{\infty} \frac{\left|a_{n}\right|^{2}}{(2 n+1)},
$$

where the first summation corresponds to the power in the electromagnetic wave and the second is the power in the acoustic wave. In order to display some important properties, (33) is rewritten in the form

where

$$
P=2 \pi \frac{\eta_{e}}{k_{e}^{2}} \sum_{n=1}^{\infty} \frac{n(n+1)}{(2 n+1)}\left|c_{n}\right|^{2}\left(1+\Delta_{n}\right),
$$

$$
\Delta_{n}=\left|\frac{a_{n}}{c_{n}}\right|^{2} \frac{1}{n(n+1)} \frac{\epsilon_{0}}{n_{0} m} \frac{k_{e}}{k_{p}} .
$$

Using (25) and noting that $\omega_{0}^{2}=n_{0} e^{2} /\left(m \epsilon_{0}\right)$, it follows that

which is a rather fundamental result.

$$
\Delta_{n}=n(n+1) \frac{\omega_{0}^{2}}{\omega^{2}} \frac{1}{\left(k_{e} a\right)\left(k_{p} a\right)} \frac{\left|\hat{K}_{n}\left(i k_{e} a\right)\right|^{2}}{\left|\hat{K}_{n}^{\prime}\left(i k_{p} a\right)-\frac{\hat{K}_{n}\left(i k_{p} a\right)}{\left(i k_{p} a\right)}\right|^{2}},
$$


It is evident from the above development that $\Delta_{n}$ is the ratio of the acoustic power in mode $n$ to the electromagnetic power in mode $n$. The result is applicable to any radiating source which produces azimuthally symmetric TM modes provided that the boundary at $r=a$ is rigid in the sense described. It is rather interesting to observe that the ratio $\Delta_{n}$ depends on three parameters, namely, (i) $k_{e} a$, which is the circumference of the rigid sphere in electromagnetic wavelengths, (ii) $k_{p} a$, which is the circumference of the rigid sphere in acoustic wavelengths, and (iii) $\omega_{0}^{2} / \omega^{2}$, the normalized plasma frequency, which is proportional to the electron density. It is worth noting that the first two parameters may be written

and

$$
\text { (i) } k_{e} a=(k a)\left(1-\frac{\omega_{0}^{2}}{\omega^{2}}\right)^{\frac{1}{2}}
$$

$$
\text { (ii) } k_{p} a=(k a)\left(1-\frac{\omega_{0}^{2}}{\omega^{2}}\right)^{\frac{1}{2}}\left(\frac{c}{u}\right) \text {, }
$$

where $k a$ is the circumference of the rigid sphere in free-space wavelengths, $u$ is the acoustic velocity, and $c$ is the velocity of electromagnetic waves in free space.

It is important to note that, everything else considered equal, $\Delta_{n}$ is proportional to $n(n+1)$. Thus, the higher order modes excite appreciably more acoustic-type radiation than the fundamental mode (i.e., $n=1$ ).

Some limiting cases are worth discussing briefly. If both $k_{e} a>>1$ and $k_{p} a>>1$, it readily follows from $(22)$ and $(23)$ that

$$
\Delta_{n} \cong n(n+1) \frac{\omega_{0}^{2}}{\omega^{2}}\left(\frac{u}{c}\right) \frac{1}{\left(k_{e} a\right)^{2}} .
$$

Since in most practical situations $u / c$ is of the order of $10^{-3}$ or $10^{-4}$, this quantity is very small, indeed.

When $k_{p} a>>1$, without any further restrictions, it is seen that

$$
\Delta_{n} \cong n(n+1) \frac{\omega_{0}^{2}}{\omega^{2}}\left(\frac{u}{c}\right) \frac{\left|\hat{K}_{n}\left(i k_{e} a\right)\right|^{2}}{\left(k_{e} a\right)^{2}},
$$

which is also small unless $\left(k_{e} a\right)$ itself is somewhat less than unity. For example, if $k_{e} a<<1$, while keeping $k_{p} a>>1$, it follows from (21) that

$$
\Delta_{n} \cong n(n+1) \frac{\omega_{0}^{2}}{\omega^{2}} \frac{u}{c} \frac{1}{\left(k_{e} a\right)^{2 n+2}}\left[\frac{(2 n) !}{n ! 2 n}\right]^{2}
$$

which may not be small, particularly for the higher order modes.

Another limiting case which is mostly of academic interest is when both $\left(k_{e} a\right)<<1$ and $\left.\left(k_{p} a\right)<<1\right)$. Then

$$
\Delta_{n} \cong \frac{n}{n+1} \frac{\omega_{0}^{2}}{\omega^{2}}\left(\frac{c}{u}\right)^{2 n+1}
$$

Since $u / c<<1$, this quantity is enormous even when $\omega / \omega_{0}$ is somewhat less than unity. Setting $n=1$ for the fundamental mode, the resulting formula for $\Delta_{1}$ is identical to that derived by Hessel and Shmoys [1962] for a point current element in a compressible plasma. From this particular limiting case, one may conclude that the formula of Hessel and Shmoys is valid only for an actual current element if its length is small compared with the wavelength of the acoustic-type wave.

\section{Excitation Considerations}

In the foregoing discussion the precise manner in which the rigid sphere is to be excited was not specified. Actually, the results derived and the conclusions drawn require only that 
the spherical surface bounding the source(s) is effectively rigid. It is also assumed, of course, that only azimuthally symmetric TM modes are to be excited. One possibility would be a rigid dielectric sphere with a radially oriented electric dipole contained within [Wait, 1964]. Another suitable method of excitation, which is considered in this paper, is to assume that the source is a perfectly conducting rigid sphere of radius $a$. At the colatitude angle $\theta_{0}$, a uniform voltage $V_{0}$ is impressed across an annular slot of infinitesimal width. In other words, the tangential electric field on the spherical surface is specified by the condition

$$
E_{\theta}(\theta, a)=\left(V_{0} / a\right) \delta\left(\theta-\theta_{0}\right),
$$

for $r=a$ and $0<\theta<\pi$, where $\delta\left(\theta-\theta_{0}\right)$ is the unit impulse function at $\theta=\theta_{0}$. The correctness of this result is confirmed by noting that

$$
\int_{0}^{\pi} E_{\theta}(\theta, a) a d \theta=\operatorname{Lim}_{\Delta \rightarrow 0} \int_{\theta_{0}-\Delta}^{\theta_{0}+\Delta} E_{\theta}(\theta, a) a d \theta=V_{0},
$$

where $2 \Delta$ is the width of the slot which is allowed to approach zero.

A general expression for the $E_{\theta}$ field in the exterior region is obtained by using (8), (16), and (17). Thus.

$$
E_{\theta}(r, \theta)=\sum_{n=0}^{\infty}\left[-\eta_{e} \frac{\hat{K}_{n}^{\prime}\left(i k_{e} r\right)}{\left(i k_{e} r\right)} c_{n}-\frac{e}{\epsilon \omega^{2} m r} \frac{\hat{K}_{n}\left(i k_{p} r\right)}{\left(i k_{p} r\right)} a_{n}\right] P_{n}^{\prime}(\cos \theta) .
$$

In the limit as $r \rightarrow a$, this result may be expressed in the form

where

$$
E_{\theta}(a, \theta)=\sum_{n=1}^{\infty} A_{n} P_{n}^{\prime}(\cos \theta)
$$

$$
A_{n}=-\eta_{e} \frac{\hat{K}_{n}^{\prime}\left(i k_{e} a\right)}{i k_{e} a} c_{n}\left(1-\delta_{n}\right)
$$

with

$$
\delta_{n}=-\frac{e}{k_{p} \omega m a}\left(\frac{a_{n}}{c_{n}}\right) \frac{\hat{K}_{n}\left(i k_{p} a\right)}{\hat{K}_{n}^{\prime}\left(i k_{e} a\right)} .
$$

Using (25), an explicit formula $\delta_{n}$ is obtained which may be written

$$
\delta_{n}=-n(n+1) \frac{\omega_{0}^{2}}{\omega^{2}} \frac{1}{\left(k_{e} a\right)\left(k_{p} a\right)} \frac{\hat{K}_{n}\left(i k_{e} a\right)}{\hat{K}_{n}^{\prime}\left(i k_{e} a\right)}\left[\frac{\hat{K}_{n}\left(i k_{p} a\right)}{\hat{K}_{n}^{\prime}\left(i k_{p} a\right)-\frac{\hat{K}_{n}\left(i k_{p} a\right)}{i k_{p} a}}\right],
$$

which is valid for the rigid boundary condition at $r=a$.

By making use of the orthogonality property of the Legendre polynomials, as indicated by (32), the coefficient $A_{n}$, from (46), may be written

$$
A_{n}=\frac{2 n+1}{2 n_{b}\left(n_{0}+1\right)} \int_{0}^{\pi} E_{\theta}(a, \theta) P_{n}^{\prime}(\cos \theta) \sin \theta d \theta .
$$

Then, as a consequence of (43),

$$
A_{n}=\frac{2 r_{s}+1}{2 n_{0}(n+1)} \frac{V_{0}}{a} P_{n}^{\prime}\left(\cos \theta_{0}\right) \sin \theta_{0} .
$$


Explicit expressions for the electromagnetic field components may now be obtained if (47) and (51) are combined with (16), (17), and (25). For example,

$$
H_{\phi}=-\frac{V_{0} \sin \theta_{0}}{2 \eta_{e} r} \sum_{n=1}^{\infty} \frac{\left(2 n_{0}+1\right)}{n_{b}(n+1)} \frac{\hat{K}_{n}\left(i k_{e} r\right)}{\hat{K}_{n}^{\prime}\left(i k_{e} a\right)} \frac{P_{n}^{\prime}\left(\cos \theta_{0}\right)}{1-\delta_{n}} P_{n}^{\prime}(\cos \theta),
$$

is an exact formula for the magnetic field which has only a $\phi$ component. In the far zone, where $\left|k_{e} r\right|>>1$, this simplifies slightly to

$$
H_{\phi} \cong-\frac{V_{0} \sin \theta_{0} e^{-i k_{e} r}}{2 \eta_{e} r} \sum_{n=1}^{\infty} \frac{\left(2 r_{0}+1\right)}{n(n+1)} \frac{P_{n}^{\prime}\left(\cos \theta_{0}\right) P_{n}^{\prime}(\cos \theta)}{\left(1-\delta_{n}\right) \hat{K}_{n}^{\prime}\left(i k_{e} a\right)},
$$

which is a spherical wave behaving like $e^{-i k_{e} \tau / r}$. In the far zone, the $\theta$ component of the electric field is obtained from

$$
E_{\theta} \cong \eta_{e} H_{\phi} \text { where } \eta_{e}=\mu_{0} \omega / k_{e}=120 \pi\left(1-\frac{\omega_{0}^{2}}{\omega^{2}}\right)^{-1 / 2} \text {. }
$$

The total power $P_{e}$ in the electromagnetic wave is obtained from

$$
P_{e}=\frac{1}{2} \operatorname{Re} \operatorname{Lim}_{r \rightarrow \infty} \int_{0}^{\pi} E_{\theta} H_{\phi}^{*}\left(2 \pi r^{2}\right) \sin \theta d \theta
$$

which is evaluated to give

$$
P_{e}=\frac{\pi}{2} \frac{V_{0}^{2}}{\eta_{e}} \sin ^{2} \theta_{0} \sum_{n=1}^{\infty} \frac{(2 n+1)}{n(n+1)} \frac{\left[P_{n}^{\prime}\left(\cos \theta_{0}\right)\right]^{2}}{\left|1-\delta_{n}\right|^{2}\left|\hat{K}_{n}^{\prime}\left(i k_{e} a\right)\right|^{2}} .
$$

Referring back to (34), it is evident that the total power radiated, $P$ (electromagnetic and acoustic), may be written as the sum

$$
P=P_{e}+P_{p}
$$

where the radiated power in the acoustic wave is given by

$$
P_{p}=\frac{\pi}{2} \frac{V_{0}^{2}}{\eta_{e}} \sin ^{2} \theta_{0} \sum_{n=1}^{\infty} \frac{(2 n+1)}{n(n+1)} \Delta_{n} \frac{\left[P_{n}^{\prime}\left(\cos \theta_{0}\right)\right]^{2}}{\left|1-\delta_{n}\right|^{2} \mid\left(\left.\hat{K}_{n}^{\prime}\left(i k_{e} a\right)\right|^{2}\right.},
$$

where $\Delta_{n}$ is given by (36).

As an important check on this derivation for the total power $P$, the Poynting vector may also be integrated over the area of the annular slot. This latter method which is related to the so-called "emf method" in antenna theory [e.g., Wait, 1959], is not very rigorous but it does lead to correct results if applied with care. Therefore, the power $P$ should also be obtained directly from

This may be written

$$
P=\frac{1}{2} \int_{\text {Area of slot }} \operatorname{Re}\left[E_{\theta} H_{\phi}^{*}\right]_{r=a} d a
$$

$$
\begin{aligned}
P & =\frac{1}{2} \operatorname{Lim}_{\Delta \rightarrow 0} \int_{\theta_{0}-\Delta}^{\theta_{0}+\Delta} \operatorname{Re}\left[E_{\theta} H_{\phi}^{*}\right]_{r=a}\left(2 \pi a^{2} \sin \theta_{0}\right) d \theta \\
& =\pi a \sin \theta_{0} V_{0} \operatorname{Re}\left(H_{\phi}\right)_{r=a},
\end{aligned}
$$

where $V_{0}$ is the voltage across the slot (which may be regarded as real). Using (52), it is seen that according to this approach

$$
P=-\frac{\pi \sin ^{2} \theta_{0}}{2 \eta_{e}} V_{0}^{2} \sum_{n=1}^{\infty} \frac{(2 n+1)}{n(n+1)}\left[P_{n}^{\prime}\left(\cos \theta_{0}\right]^{2} \operatorname{Re}\left[\frac{\hat{K}_{n}\left(i k_{e} a\right)}{\hat{K}_{n}^{\prime}\left(i k_{e} a\right)\left(1-\delta_{n}\right)}\right] .\right.
$$


The exact correspondence between (57) and (62) is a result of the identity

$$
\operatorname{Re}\left[\frac{\hat{K}_{n}\left(i k_{e} a\right)}{\hat{K}_{n}^{\prime}\left(i k_{e} a\right)} \frac{1}{1-\delta_{n}}\right]=-\frac{1+\Delta_{n}}{\left|\hat{K}_{n}^{\prime}\left(i k_{e} a\right)\right|^{2}\left|1-\delta_{n}\right|^{2}},
$$

where $\Delta_{n}$ and $\delta_{n}$ are defined by (36) and (49), respectively. This identity is verified without difficulty, if it is remembered that,

for $z$ real.

$$
\operatorname{Re} \hat{K}_{n}(i z)\left[\hat{K}_{n}^{\prime}(i z)\right]^{*}=-1
$$

Equation (62) may be used for the power calculation for a slotted sphere of arbitrary size immersed in a compressible plasma. When $k_{e} a$ and $k_{p} a$ are not large compared with unity, the series converges rapidly. Then, it is of interest to see that the power in the first mode may be written

where, according to (36),

$$
P \cong \frac{3 \pi \sin ^{4} \theta_{0}}{4 \eta_{e}} V_{0}^{2} \frac{1+\Delta_{1}}{\left|1-\delta_{1}\right|^{2}}\left(k_{e} a\right)^{4}
$$

and according to (49),

$$
\Delta_{1} \cong 2 \frac{\omega_{0}^{2}}{\omega^{2}} \frac{1}{\left(k_{e} a\right)^{3}} \frac{1}{\left(k_{p} a\right)\left|\hat{K}_{1}^{\prime}\left(i k_{p} a\right)-\frac{\hat{K}_{1}\left(i k_{p} a\right)}{\left(i k_{p} a\right)}\right|^{2}},
$$

$$
\delta_{1} \cong i \frac{\omega_{0}^{2}}{\omega^{2}} \frac{2}{\left(k_{p} a\right)}\left[\frac{\hat{K}_{1}\left(i k_{p} a\right)}{\hat{K}_{1}^{\prime}\left(i k_{p} a\right)-\frac{\hat{K}_{1}\left(i k_{p} a\right)}{i k_{p} a}}\right]
$$

where it should be remembered that

$$
k_{p} a=(c / u) k_{e} a
$$

If $u / c$ is sufficiently large that $k_{p} a>>1$, while still maintaining $k_{e} a<<1$, the above formulas for $\Delta_{1}$ and $\delta_{1}$ simplify to

and

$$
\Delta_{1} \cong 2 \frac{\omega_{0}^{2}}{\omega^{2}} \frac{u}{c} \frac{1}{\left(k_{e} a\right)^{4}}
$$

$$
\delta_{1} \cong-2 i \frac{\omega_{0}^{2}}{\omega^{2}} \frac{u}{c} \frac{1}{k_{e} a} .
$$

Using the above result, it is possible to write a simple formula for the ratio of the total power $P$ for a compressible plasma when $u / c$ is finite to the total power $P_{0}$ for an incompressible plasma (such that $u / c=0)$. Thus, by using (65), (69), and (70), it is seen that, for $n=1$,

$$
\frac{P}{P_{0}} \cong \frac{1+2 \frac{\omega_{0}^{2}}{\omega^{2}} \frac{u}{c} \frac{1}{\left(k_{e} a\right)^{4}}}{1+4 \frac{\omega_{0}^{4}}{\omega^{4}}\left(\frac{u}{c}\right)^{2} \frac{1}{\left(k_{e} a\right)^{2}}}
$$

For this same condition, it is worth noting that the ratio of the power $P_{e}$ of the electromagnetic wave in the compressible plasma to the total power for the incompressible plasma, is given by

$$
\frac{P_{e}}{P_{0}} \cong \frac{1}{1+4 \frac{\omega_{0}^{4}}{\omega^{4}}\left(\frac{u}{c}\right)^{2}} \frac{1}{\frac{1}{\left(k_{e} a\right)^{2}}} .
$$


As indicated previously, the ratio of the acoustic power $P_{p}$ to the power electromagnetic $P_{e}$, for $n=1$, is

$$
\frac{P_{p}}{P_{e}} \cong 2 \frac{\omega_{0}^{2}}{\omega^{2}} \frac{u}{c} \frac{1}{\left(k_{e} a\right)^{4}}
$$

for these same conditions (i.e., $k_{e} a<<1$ and $k_{p} a>>1$ ).

\section{Concluding Remarks}

On the basis of the analysis in this paper, it would appear that an electron-acoustic wave will be excited by a slotted-sphere antenna which is immersed in a compressible plasma. However, if this effect is to be measurable, the operating wave frequency must be an appreciable fraction of the plasma frequency. It also appears that the relative power in the acoustic wave is increased as the overall dimensions of the antenna are reduced.

\section{References}

Chen, Kun-Mu (Jul. 9-11, 1962), Interaction of a radiating source with a plasma-effect of an electroacoustic wave, Program Digest, Symposium on Antennas and Propagation, Boulder, Colo., 252-257.

Hessel, A., N. Marcuvitz, and J. Shmoys (Jan. 1962), Scattering and guided waves at an interface between air and a compressible plasma, IRE Trans. AP-10, $48-54$.

Hessel, A., and J. Shmoys (1962), Excitation of plasma waves in a homogeneous isotropic plasma by a dipole, Proc. Symposium on Electromagnetic and Fluid Dynamics af Gaseous Plasma, 173-184 (Polytechnic Press, Brooklyn, N.Y.)

Morse, P. M., and H. Feshbach (1953), Methods of theoretical physics, Pt. II (McGraw-Hill Book Co., New York).

Oster, L. (Jan. 1960), Linearized theory of plasma oscillations, Rev. Mod. Phys. 32, 141-168.

Schelkunoff, S. A. (1943), Electromagnetic waves (Van Nostrand Co., Ltd., New York).

Schelkunoff, S. A. (1952), Advanced antenna theory (John Wiley \& Sons, Inc., New York, N.Y.).

Seshadri, S. R. (Jan. 1963a), Excitation of plasma waves in an unbounded homogeneous plasma by a line source, IEEE Trans. MTT-11, 39-50.

Seshadri, S. R. (Jul. 9-11, 1963b), Radiation from an electromagnetic source in a half-space of compressible plasma-surface waves, Program Digest, Symposium on Antennas and Propagation, Boulder, Colo., 275-276.

Wait, J. R. (1959), Electromagnetic radiation from cylindrical structures (Pergamon Press, London).

Wait, J. R. (1964), Radiation from sources in compressible plasma media, Can. J. Pbys. 42 (in press).

Whale, H. A. (Jan. 15, 1963), The excitation of electroacoustic waves by antennas in the ionosphere, J. Geophys. Res. 68, 415-422.

Whale, H. A. (Feb. 1, 1964), Ion sheath effects near antennas radiating within the ionosphere, J. Geophys. Res. 69, $447-455$.

Yildiz, A. (Dec. 1963), Scattering of plane plasma waves from a plasma sphere, Il Nuovo Cimento, Serie X, 30, $1182-1207$.

Yildiz, M., and S. N. Samaddar (1963), A note on wave phenomena due to a moving and oscillating dipole in a compressible plasma column, Can. J. Phys. 41, 2268-2275.

(Paper 68D10-412) 